\title{
Monitoring Dissolution Rate of Amiodarone Tablets by a Multiple Fiber-Optic Sensor System
}

\author{
Turghun Muhammad ${ }^{1,3}$, Jide Wang ${ }^{1}$, Mei Li-wan' ${ }^{2}$, and Jian Chen ${ }^{2}$ \\ 'College of Chemistry \& Chemical Engineering, Xinjiang University, Urumqi 830046, China \\ ${ }^{2}$ College of Pharmacy, Xinjiang Medical University, Urumqi 830054, China
}

\begin{abstract}
A fiber-optic dissolution testing (FODT) system for solid pharmaceutical formulations has been constructed. The system is based on a charge-coupled device (CCD) detector and includes six fiber-optic probes. Light is produced by a small deuterium arc lamp illuminating an optical fiber bundle. Six fiber-optic dipping probes were constructed with reflection geometry. The light passes back and forth through the flow-through cuvette backed by a coated aluminum mirror. The sampling interval was typically $30 \mathrm{sec}$ for all probes.

The system was tested for different tablet and capsule formulations. Amiodarone is a Class III anti-arrhythmic agent. It is used for treatment of many ventricular and supraventricular arrhythmias, including arterial fibrillation. It is a highly hydrophobic drug, and factors of its formulation, including constitutional heterogeneity, surface membrane, and production technology, influence its absorption. The dissolution of marketed amiodarone tablets was tested by the system, and dissolution parameters were directly obtained from the dissolution curves. It was found that there were significant differences in the dissolution of these products.
\end{abstract}

\section{INTRODUCTION}

Dissolution testing of pharmaceutical preparations is performed for quality control purposes and to obtain information about the rate of release of active compounds into the human body (1). This is normally done in vitro, but by choosing the experimental parameters with care, a good in vivo correlation can be reached. The standard methods for dissolution testing of pharmaceutical preparations are both time-consuming and labor-intensive. The typical procedure involves dissolution of the tablets in glass vessels under heating and agitation of the dissolution medium. The agitation speed and the amount and composition of the medium can be chosen to fit the in vivo correlation. Additionally, the physical parameters, such as the shape and position of the paddle and the vessels, are strictly regulated by pharmacopeia guidelines. Samples are withdrawn from the vessels at selected times, filtered, and analyzed by HPLC or spectrophotometry. As an alternative, automatic flow-through sampling can be used in combination with a spectrometer. However, these methods suffer from several disadvantages. The overall analysis time is long. Furthermore, the total number of samples is limited by both the sampling time (e.g., 0.5-1 $\mathrm{min}$ ) and the sampling volume. Repeated large sampling volumes will decrease the total vessel volume, which may affect results. Consequently, for a six-sample dissolution test, samples may be obtained at a minimum of five-minute intervals. Another drawback of the automatic flow-through spectrometer is that some substances adsorb to the tubes,

${ }^{3}$ Corresponding author. and it is difficult to clean them after use. Foaming can occur when surfactants are used in the dissolution medium.

A different approach is to perform the measurements by in situ probing rather than by extracting samples from the vessels. The use of fiber optics has previously been reported in connection with dissolution testing of pharmaceutical preparations. Single-probe systems were developed for on-line fiber-optic spectroscopy in dissolution testing $(2,3)$. Later, multi-probe systems (4-8) were reported. A 12-probe fiber-optic dissolution testing system with dual-path designed to extend the analyte concentration range has been reported (9), but it has difficulty in covering large concentration ranges.

In this paper we report on a dissolution testing system with six fiber-optic probes. There are a series of probe tips that have light paths of $1.0,2.0,4.0,6.0$, and $10.0 \mathrm{~mm}$. The analyte concentration determines which tip may be used. The system is very useful for investigating drugs from different manufacturing facilities. Several articles investigated the dissolution testing of amiodarone tablets after it was reported that imported amiodarone products had higher therapeutic efficacy than domestic products (10-14). Therefore, we used the FODT system to investigate dissolution of amiodarone tablets on the domestic market as an example.

\section{MATERIAL AND METHODS \\ Fiber-Optic Dissolution System}

The dissolution testing system is shown in Figures 1 and 2. It consists of a deuterium $\left(D_{2}\right)$ lamp, six fiber-optic dipping probes, and a CCD (Charge Coupled Device) detector. The $\mathrm{D}_{2}$ lamp (Heraeus Noblelight GmbH, Hanau, 


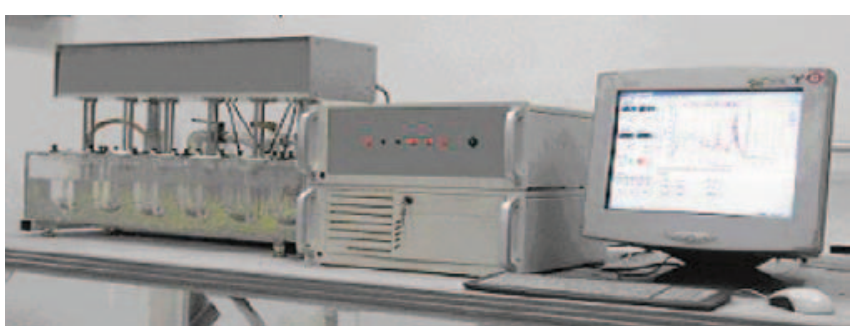

Figure 1. Fiber-optic dissolution testing system.

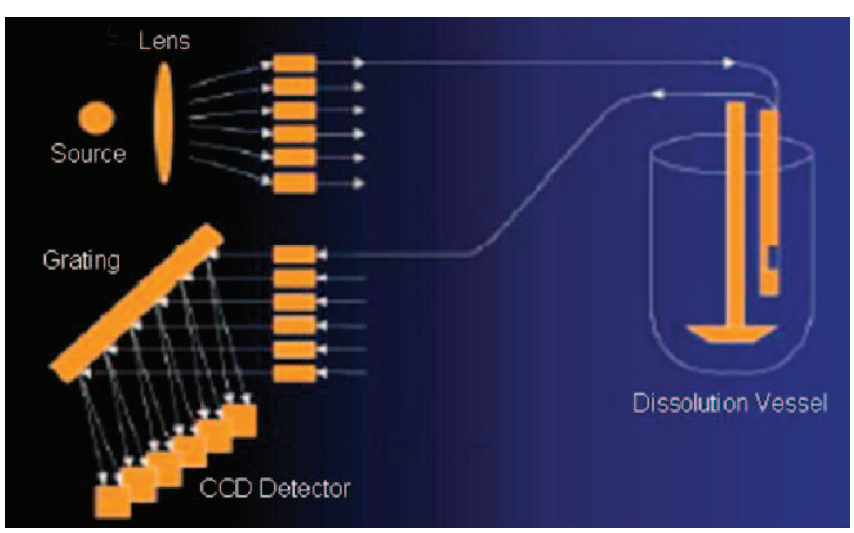

Figure 2. Optical pathway scheme of the fiber-optic dissolution system.

Germany) was a 30-W, small arc (0.5-mm) lamp mounted in a lamp house with optics for coupling into optical fibers. The probe head, shown in Figure 3, includes an illumination fiber, an Al-coated mirror, and two detection fibers all mounted in stainless steel cylinders. The light path can be altered to $1.0,2.0,4.0,6.0$, or $10.0 \mathrm{~mm}$ by substituting the far end of the probe head for another one.

\section{Software}

A custom software package was used for data transfer and communication with the CCD, and for data calculation and presentation. Instrumental settings such as analytical

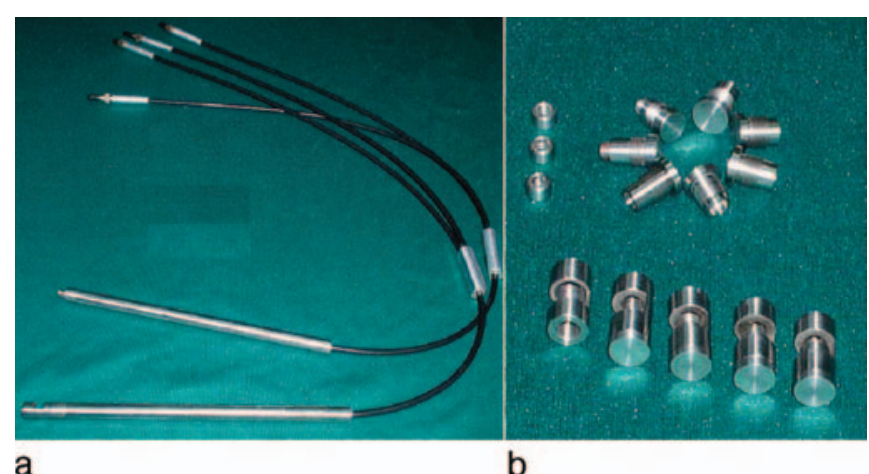

b

Figure 3. Photograph showing details of the dissolution testing system. (a) Dipping probe version, two probes and (b) disassembled probe heads. wavelength, sampling time, and sampling interval are set, and dissolution curves are displayed live on the screen for all probes simultaneously. The software includes graphical routines for calibration against blank and reference solutions. The dissolution test can be executed using one or more analytical wavelengths together with a background wavelength.

\section{Reference Method}

Reference measurements were normally performed using a spectrophotometer (Citro40, Hewlett Packard, GBC Scientific Equipment Pty Ltd, Australia). Samples were extracted from the dissolution vessels and filtered through 0.80- $\mu \mathrm{m}$ filters (MFS-25 CA 0.80, Shanghai Bandao Industry Co., Ltd., Shanghai, China) prior to measurements in a 1-cm quartz cuvette according to Chinese Pharmacopoeia (ChP) (15).

\section{Chemicals}

The water was prepared by degassing redistilled water. Sodium lauryl sulfate (SLS) solution (0.25\%) was prepared from SLS obtained from Xi'an Chemical Reagent Co., Ltd. (Xi'an, China). Methanol (analytical reagent) was obtained from Xi'an Chemical Reagent Co., Ltd. (Xi'an, China). Amiodarone hydrochloride was obtained from Sigma-Aldrich (USA).

\section{Tablets}

Several samples of 200-mg amiodarone hydrochloride tablets were obtained on the local market: $\mathrm{H}$ (Cordarone, Hangzhou Sanofi-Synthelabo Minsheng Pharmaceutical Co., Ltd., Lot. 062, production date: 05.08.19); B (Lot.050501, production date: 05.05.31);W (Lot.05050601, production date: 05.05.04); J (Lot.030703, production date:03.07); and S (050902, production date: 05.07). Lots B, W, J, and S are representative of domestic pharmaceutical corporations.

\section{Amiodarone Calibration Standard Solutions}

A stock solution of amiodarone was prepared by dissolving $25 \mathrm{mg}$ of the analyte in $25 \mathrm{~mL}$ of methanol. The stock solution of amiodarone was diluted with $0.25 \%$ SLS to obtain calibration solutions of $40,80,120,160,200$, and $240 \mathrm{mg} / \mathrm{L}$, corresponding to cumulative dissolution concentrations of $18.0,36.0,54.1,72.1,90.1$, and $108.1 \%$, respectively.

\section{Dissolution Testing}

In situ FODT system was turned on without light to correct the dark current and potential ambient light interferences. The six probes were dipped automatically into the dissolution vessels, which were each filled with $900 \mathrm{~mL}$ of $0.25 \% \mathrm{SLS}$ solution and equilibrated to $37.0^{\circ} \mathrm{C}$. The lamp was then turned on to get absorbances of blank solutions. Using the paddle method at a stirring speed of $100 \mathrm{rpm}$, six $\mathrm{H}$ tablets were dropped, one into each vessel. The detection and reference wavelengths were set at 
$305 \mathrm{~nm}$ and $550 \mathrm{~nm}$, respectively. The ending time can be set at any time for automatic operation. The dissolution test was then initiated, and the dissolution curves for up to six vessels were followed in "live" mode on the computer screen. The dissolution data were calculated according to calibration curves.

For the ChP reference method, sample aliquots were collected and filtered after 3,6, 9, 12, and $20 \mathrm{~min}$. The absorbances were measured at $305 \mathrm{~nm}$ after the dissolution experiment.

\section{RESULTS AND DISCUSSION Calibration Curves}

\section{Selection of Detection Wavelength}

According to the UV absorption spectra of amiodarone calibration standard solutions (Figure 4), there are two absorption maxima at 242 and $305 \mathrm{~nm}$. But at $242 \mathrm{~nm}$, the absorbance is too high, and interference is more likely than at $305 \mathrm{~nm}$. The calibration curve was constructed by linear regression using the absorbance of amiodarone at $305 \mathrm{~nm}$ plotted against the corresponding concentrations expressed by cumulative dissolution (\%).

$$
A=0.0199 C-0.0333 \quad r=0.9996
$$

$A$ corresponds to absorbance and $C$ corresponds to the concentration ( $n=6)$. Very good linearity according to Beer-Lambert law was achieved at $305 \mathrm{~nm}$ in the concentration range of 18.0-108.1\% (corresponding to $40.00-240.00 \mathrm{mg} / \mathrm{L}$ ), so $305 \mathrm{~nm}$ was selected as the detection wavelength for the FODT system.

\section{Selection of Light Path}

The FODT system was started by acquiring a background image with the lamp off, which corrects for the dark current and for potential ambient light interferences. To choose the most appropriate light path, probes with light paths of 1.0,2.0,4.0,6.0, and $10.0 \mathrm{~mm}$ were dipped in a blank solution and the series of amiodarone standard calibration solutions. Excellent

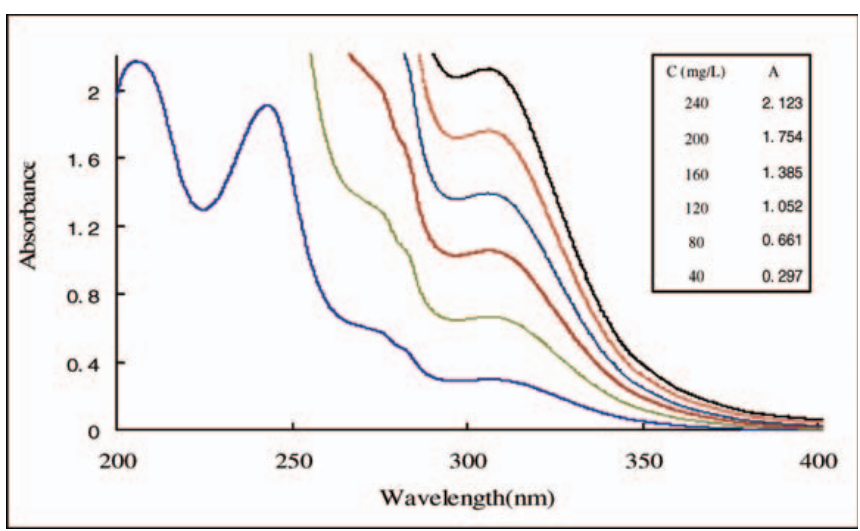

Figure 4. UV absorption spectra of amiodarone calibration standard solutions and absorptions at $305 \mathrm{~nm}$ obtained by GBC spectrophotometer. linearity was achieved for concentrations ranging from 40 to $240 \mathrm{mg} / \mathrm{L}$ at $305 \mathrm{~nm}$ using the $6.0-\mathrm{mm}$ probe, so that one was chosen. The calibration curves of six channels are shown as follows:

$$
\begin{array}{ll}
\text { Channel 1: } C=92.416 A-0.0326 & r=0.99999 \\
\text { Channel 2: } C=93.135 A-0.0402 & r=0.99998 \\
\text { Channel 3: } C=94.836 A-0.4033 & r=0.99994 \\
\text { Channel 4: } C=93.495 A-0.2582 & r=0.99997 \\
\text { Channel 5: } C=91.680 A-0.0324 & r=0.99999 \\
\text { Channel 6: } C=92.778 A-0.3108 & r=0.99998
\end{array}
$$

$C$ corresponds to the concentration expressed by cumulative dissolution (\%) and $A$ corresponds to absorbance $(n=7)$.

\section{Comparison of Two Methods}

Dissolution data for $\mathrm{H}$ amiodarone tablets obtained by the FODT method and the ChP reference method are shown in Table 1 and demonstrate good agreement between the two methods.

Tablet solutions (concentration of about $40 \mathrm{mg} / \mathrm{L}$ ) were spiked $20.0 \mathrm{~mL}$ into each of 12 measuring flasks and separated into four groups, one of which served as background. Into the other three groups was added 2.5 $\mathrm{mL}$ of amiodarone stock solution at concentrations of 40 , 120 , and $200 \mathrm{mg} / \mathrm{L}$ to give amiodarone solutions at three different concentrations. Absorbances were determined five times at 10-min intervals by FODT. The accuracy was calculated and found to be $97.8 \%, 97.37 \%$, and $99.1 \%$; RSDs were $1.0 \%, 0.6 \%$, and $0.7 \%(n=5)$, respectively.

\section{Dissolution Curves}

In conventional dissolution testing, the tedious process of analyzing the sample involves sample filtration before UV analysis, which is very labor intensive, especially when comparing the dissolution performance of products from several factories. The outstanding feature of a fiber-optic dissolution system is the capability of acquiring continuous drug release in real time. The

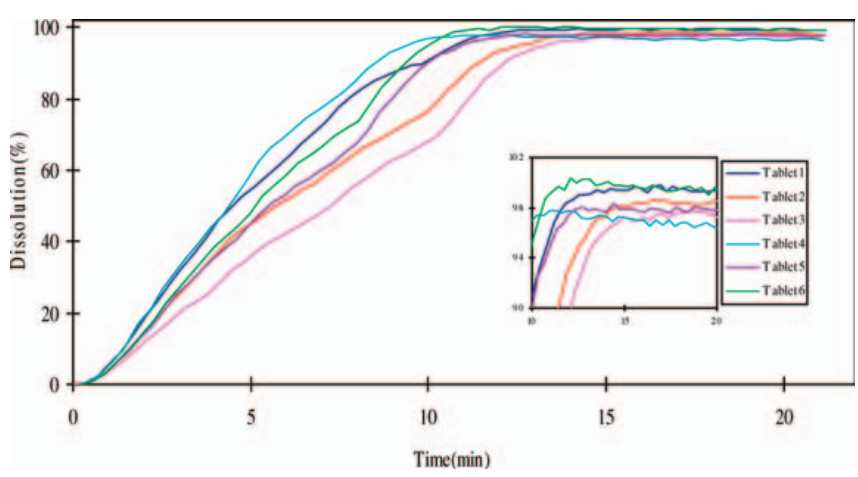

Figure 5. Dissolution profiles of six product $\mathrm{H}$ tablets. Dissolution test conditions: $900 \mathrm{~mL}$ of $0.25 \% \mathrm{SLS}$ solution at $37.0^{\circ} \mathrm{C}$ with paddles at $100 \mathrm{rpm}$. Spectra were acquired at 0.5-min intervals. 
resulting FODT dissolution curves of $\mathrm{H}$ amiodarone tablets are shown in Figure 5. ChP reference measurements were performed only for $\mathrm{H}$ amiodarone tablets; those data are listed in Table 1. In the case of $\mathrm{H}$ tablets, cumulative dissolution percentages were larger than $96 \%$ for all tablets as shown in Table 2. This occurred even though some analyte was removed from the vessels for carrying out reference testing. In addition, it can provide information on critical kinetic behaviors of the active pharmaceutical ingredient such as super-saturation, form conversion, intrinsic solubility, and also the entire kinetic process of the formulation dissolution.

In conventional methods, dissolution parameters are calculated according to models such as the Weibull analysis, provided that the mass of the batch formulation is consistent with the dissolution profile. The parameters of different tablets can be readily obtained from dissolution curves or from the corresponding data in this system. As shown in Table 3, the mean T30\%, T50\%, Td\%, and $\mathrm{T} 80 \%$ values of $\mathrm{H}$ tablets are lower than those of tablets from the other factories, except for the mean IT value (2.78 min), which is near the 2.20 -min value of $\mathrm{J}$ tablets. Figure 5 clearly shows that more than $95 \%$ of the labeled amount of the drug substance in $\mathrm{H}$ tablets was dissolved within $15 \mathrm{~min}$, so it may be defined as a rapidly dissolving, immediate-release formulation.

Table 1. Comparison of the Percentage of H Amiodarone Tablets Dissolution between FODT and ChP Methods ( $n=6)$.

\begin{tabular}{llcc}
\hline \multirow{2}{*}{ Time $(\mathrm{min})$} & \multicolumn{3}{l}{ Percentage of amiodarone dissolution $(\% \pm \mathrm{SD})$} \\
& FODT & ChP & P \\
\hline 3 & $27.1 \pm 4.1$ & $35.2 \pm 5.5$ & $>0.05$ \\
\hline 6 & $56.4 \pm 6.5$ & $63.4 \pm 7.4$ & $>0.05$ \\
\hline 9 & $80.2 \pm 7.4$ & $82.0 \pm 7.0$ & $>0.05$ \\
\hline 12 & $95.9 \pm 4.4$ & $94.0 \pm 2.4$ & $>0.05$ \\
\hline 20 & $98.1 \pm 1.3$ & $96.3 \pm 1.4$ & $>0.05$ \\
\hline
\end{tabular}

Table 2. Cumulative Dissolution of Tablets of Different Factories in $80 \mathrm{~min}$ (\%). ${ }^{\mathrm{a}}$

\begin{tabular}{|c|c|c|c|c|c|c|c|}
\hline \multirow{2}{*}{ Factory } & \multicolumn{6}{|c|}{ Tablet } & \multirow{2}{*}{ Mean \pm SD } \\
\hline & 1 & 2 & 3 & 4 & 5 & 6 & \\
\hline $\mathrm{H}$ & 99.36 & 97.88 & 97.44 & 96.38 & 97.65 & 99.33 & $98.00 \pm 1.06$ \\
\hline B & 92.62 & 92.94 & 94.80 & 94.35 & 93.48 & 94.44 & $93.77 \pm 0.81$ \\
\hline S & 88.37 & 87.06 & 87.14 & 85.45 & 85.87 & 88.51 & $87.07 \pm 1.14$ \\
\hline J & 85.75 & 83.76 & 84.19 & 86.39 & 85.10 & 85.44 & $85.11 \pm 0.90$ \\
\hline W & 81.56 & 87.04 & 76.94 & 85.70 & 83.81 & 85.40 & $83.41 \pm 3.36$ \\
\hline
\end{tabular}

${ }^{a}$ For $\mathrm{H}$ and $\mathrm{B}$ tablets, the dissolution times were $21 \mathrm{~min}$ and $60 \mathrm{~min}$, respectively.
B tablets had the best precision between tablets with the lowest SD $(\leq 0.57)$ for all parameters. More than $90 \%$ of the labeled amount of the drug substance was dissolved within $15 \mathrm{~min}$. This is comparable to the $\mathrm{H}$ tablets; consequently, the $B$ tablets may also be considered a rapidly dissolving, immediate-release formulation. The average amount of drug substance dissolved within 60 minutes was $93.77 \%$, which is $3.23 \%$ less than the amount of $\mathrm{H}$ tablets that dissolved within only $21 \mathrm{~min}$, as shown in Table 2. This means that B tablets have lower bioavailability than $\mathrm{H}$ tablets.

$\mathrm{S}$ and $\mathrm{J}$ tablets presented similar dissolution curves and had close dissolution parameters except for IT ( $S=5.47$, $\mathrm{J}=2.20$ ), as shown in Table 3. Their percentages of cumulative dissolution were higher than those of $\mathrm{W}$ tablets within $80 \mathrm{~min}$, but they are still much lower than those of $\mathrm{H}$ and $\mathrm{B}$ tablets. The mean $\mathrm{T} 80 \%$ values of $\mathrm{S}, \mathrm{J}$, and $\mathrm{W}$ tablets are $40.24 \mathrm{~min}, 47.14 \mathrm{~min}$, and $62.31 \mathrm{~min}$, respectively, which are much longer than those of $\mathrm{H}$ and $\mathrm{B}$ tablets. W tablets especially had a very long T80\% value, so these may not be considered immediate-release form. There are significant differences among the amiodarone tablets from these 5 factories.

Being a fully automatic process, FODT does not introduce human error, so the comparison result is more representative than the manual process. This system will show potential application in the testing of rapidly disintegrating tablets with its speed, and in sustained-release preparations with its ability to perform with great stability over several days or even weeks.

\section{CONCLUSIONS}

Fiber-optic dissolution testing was proven useful for pharmaceutical solid formulations. Simultaneous data registration was obtained with parallel detection. Furthermore, the novel design, having a series of light paths of 1.0,2.0,4.0,6.0, and $10.0 \mathrm{~mm}$, extended the concentration range to most of the required range. With a 305-nm detection wavelength and a probe head of 6.0$\mathrm{mm}$ light path, this system produced amiodarone dissolution testing results with very good linearity over the whole concentration range. It also yielded high accuracy. The system was used to investigate amiodarone tablets from different factories on the local market. $\mathrm{H}$ and $\mathrm{B}$ tablets had much faster and coherent dissolution profiles than others and can be considered as immediate-release form; $\mathrm{S}$ and $\mathrm{J}$ had similar dissolution parameters, and their dissolution times were much longer than those of $\mathrm{H}$ and $\mathrm{B}$ tablets; the $W$ tablets had a very low dissolution rate that was five times lower than that of $\mathrm{H}$ and $\mathrm{B}$ tablets. These significant differences among the products from different factories indicate that the dissolution of the product should be tested to control the quality and ensure clinical efficacy. The in situ system proved to be productive, useful, and labor saving and yielded much more information than conventional methods. 
Table 3. Dissolution Parameters ( $\mathrm{min}$ ) of $\mathrm{H}, \mathrm{B}, \mathrm{S}, \mathrm{J}$, and W Amiodarone Tablets in Dissolution Media. ${ }^{\mathrm{a}}$

\begin{tabular}{|c|c|c|c|c|c|c|c|}
\hline \multirow{2}{*}{ Parameter } & \multicolumn{6}{|c|}{ Tablets } & \multirow{2}{*}{ Mean \pm SD } \\
\hline & 1 & 2 & 3 & 4 & 5 & 6 & \\
\hline$r$ & 0.99999 & 0.99998 & 0.99994 & 0.99997 & 0.99999 & 0.99998 & \\
\hline \multicolumn{8}{|l|}{$\mathbf{H}$} \\
\hline T30\% & 2.87 & 3.42 & 4.3 & 2.79 & 3.43 & 3.24 & $3.34 \pm 0.54$ \\
\hline T50\% & 4.54 & 5.74 & 7.22 & 4.36 & 5.58 & 5.14 & $5.43 \pm 1.03$ \\
\hline Td\% & 5.97 & 7.77 & 9.1 & 5.37 & 7.42 & 6.54 & $7.03 \pm 1.35$ \\
\hline Т80\% & 7.81 & 10.31 & 11.16 & 7.34 & 8.99 & 8.46 & $9.01 \pm 1.47$ \\
\hline IT & 2.72 & 2.75 & 3.14 & 2.52 & 2.9 & 2.62 & $2.78 \pm 0.22$ \\
\hline \multicolumn{8}{|l|}{ B } \\
\hline T30\% & 3.83 & 3.89 & 3.9 & 3.77 & 3.57 & 3.77 & $3.79 \pm 0.12$ \\
\hline T50\% & 5.67 & 5.83 & 6.21 & 5.49 & 5.35 & 5.5 & $5.67 \pm 0.31$ \\
\hline Td\% & 7.11 & 7.38 & 7.55 & 6.79 & 6.71 & 6.86 & $7.06 \pm 0.34$ \\
\hline T80\% & 9.69 & 9.98 & 10.13 & 8.95 & 8.83 & 9.06 & $9.44 \pm 0.57$ \\
\hline IT & 5.06 & 5.14 & 4.19 & 4.75 & 4.8 & 4.35 & $4.71 \pm 0.38$ \\
\hline \multicolumn{8}{|l|}{ S } \\
\hline T30\% & 5.53 & 5.64 & 8.22 & 7.4 & 6.66 & 6.26 & $6.62 \pm 1.04$ \\
\hline T50\% & 9.18 & 9.55 & 12.02 & 11.49 & 10.99 & 10.13 & $10.56 \pm 1.12$ \\
\hline Td\% & 14.32 & 15.06 & 17.47 & 17.65 & 17.2 & 15.12 & $16.14 \pm 1.46$ \\
\hline T80\% & 34.91 & 39.08 & 41.15 & 46.74 & 45.28 & 34.27 & $40.24 \pm 5.17$ \\
\hline IT & 4.05 & 4.13 & 8.22 & 6.25 & 4.8 & 5.36 & $5.47 \pm 1.58$ \\
\hline \multicolumn{8}{|l|}{ J } \\
\hline T30\% & 3.02 & 3.55 & 3.66 & 3.38 & 3.48 & 3.54 & $3.44 \pm 0.23$ \\
\hline T50\% & 6.15 & 7.43 & 6.6 & 6.54 & 7.15 & 6.91 & $6.80 \pm 0.46$ \\
\hline Td\% & 12.01 & 14.69 & 13.22 & 12.48 & 14.22 & 13.38 & $13.33 \pm 1.01$ \\
\hline T80\% & 43.04 & 53.18 & 50.72 & 42.22 & 48.31 & 45.35 & $47.14 \pm 4.36$ \\
\hline IT & 2.04 & 2.12 & 2.18 & 2.23 & 2.29 & 2.34 & $2.20 \pm 0.11$ \\
\hline \multicolumn{8}{|l|}{$\mathbf{w}$} \\
\hline T30\% & 6.08 & 6.79 & 8.82 & 5.65 & 5.51 & 10.14 & $7.16 \pm 1.89$ \\
\hline T50\% & 13.33 & 13.11 & 18.41 & 11.92 & 13.07 & 16.9 & $14.46 \pm 2.57$ \\
\hline Td\% & 25.9 & 22.95 & 36.78 & 22.01 & 24.9 & 27.2 & $26.62 \pm 5.32$ \\
\hline T80\% & 71.71 & 53.68 & - & 54.8 & 71.44 & 59.94 & $62.31 \pm 8.78$ \\
\hline IT & 4.05 & 4.63 & 6.71 & 2.23 & 2.79 & 9.38 & $4.96 \pm 2.67$ \\
\hline
\end{tabular}

a $\mathrm{Tx} \%$ : time corresponding to the percentage (x\%) of amiodarone released from the tablet; Td\%: time corresponding to $63.2 \%$ of amiodarone released from the tablet; r: correlation coefficient $(n=6)$; IT: inflexion time (the time from which dissolution rate began to decrease). 


\section{ACKNOWLEDGMENT}

This research was supported by the National Natural Science Foundation of China (Nos. 20765005).

\section{References}

1. United States Pharmacopeia and National Formulary USP 24-NF19; United States Pharmacopeial Convention, Inc.: Rockville, MD, 2000; p 1941.

2. Schatz, C.; Ulmschneider, M.; Altermatt, R.; Marrer, S.; Altorfer, H. Manual In Situ Fiber Optic Dissolution Analysis in Quality. Dissolution Technol. 2000, 7 (2), 6-13.

3. Brown, C.W.; Lin, J. Interfacing a Fiber-Optic Probe to a Diode Array UV-Visible Spectrophotometer for Drug Dissolution Tests. Appl. Spectrosc. 1993, 47, 615-618.

4. Aldridge, P. K.; Melvin, D. W.; Williams, B. A.; Bratin, K.; Kostek, L. J.; Sekulic, S. S. A robotic dissolution system with on-line fiber-optic UV analysis. J. Pharm. Sci. 1995, 84 (8), 909-914.

5. Schatz, C.; Ulmschneider, M.; Altermatt, R.; Marrer, S.; Altorfer, $\mathrm{H}$.; Thoughts on Fiber-Optics in Dissolution Testing. Dissolution. Technol. 2001, 8 (2), 6-13.

6. Nir, I.; Johnson, D.; Johansson, J.; Schatz, C. Application of Fiber optic Dissolution Testing for Actual Products. Pharm. Technol. 2001, 25, 33-40.

7. Cho, J.H.; Gemperline, P.J.; Salt, A.; Walker, D. S. UV-Visible Spectral Dissolution Monitoring by In Situ Fiber-Optic Probes. Anal. Chem. 1995, 67 (17), 2858-2863.

8. Gemperline, P. J.; Cho, J. H.; Baker, B.; Batchelor, B.; Walker, D.S. Determination of multicomponent dissolution profiles of pharmaceutical products by in situ fiber-optic UV measurements. Anal. Chim. Acta 1997, 345, 155-159.

9. Johansson, J.; Cauchi, M.; Sundgren, M. Multiple fiber-optic dual-beam UV/Vis system with application to dissolution testing. J. Pharm. Biomed. Anal. 2002 29 (3), 469-476.

10. Jin-Hong, H.; Zhen, L.; Li-Chao, Z.; Jing, S. Pharmaceutical quality study on amiodarone hydrochloride: I. In vitro dissolution rate of market tablets. Acad.J. Second Mil. Med. Univ. 2000, 21 $140-142$.

11. Stefan, R.-I.; Aboul-Enein, H. Y.; Baiulescu, G.-E. Amiodarone-selective membrane electrode. Sens. Actuators 1996, 37 (3), 141-144.

12. Rong-Sheng, T. Study on dissolution of amiodarone hydrochloride tablets. Herald of Med. 2001, 20, 244-245.

13. Meng, X.; Mojaverian, P.; Doedée, M.; Lin, E.; Weinryb, I.; Chiang, S.T.; Kowey, P. R. Bioavailability of amiodarone tablets administered with and without food in healthy subjects. Am. J. Cardiol. 2001, 87, 432-435.

14. Zi-Xuan, Y. Dissolution testing method of amiodarone hydrochloride tablets. China Pharma. 2005, 14, 30-31.

15. Chinese Pharmacopoeia; Chemical Industry Press: Beijing, China, 2005; Vol. Il, p 73. 